\title{
(6) OPEN ACCESS Prognostic significance of foveal capillary drop-out
and previous panretinal photocoagulation for
diabetic macular oedema treated with ranibizumab
}

\author{
Andreas Ebneter, Sebastian Wolf, Martin S Zinkernagel
}

\begin{abstract}
- Additional material is published online only. To view please visit the journal online (http://dx.doi.org/10.1136/ bjophthalmol-2014-306482)
\end{abstract}

Department of Ophthalmology, Bern University Hospital and University of Bern, Bern, Switzerland

\section{Correspondence to} Dr Andreas Ebneter, Department of Ophthalmology, Inselspital, Bern $\mathrm{CH}-3010$, Switzerland;

andreas.ebneter@insel.ch, ebneter.andreas@gmail.com

Received 3 December 2014 Revised 19 June 2015 Accepted 25 June 2015 Published Online First 17 July 2015

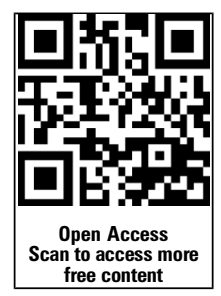

CrossMark

To cite: Ebneter $A$, Wolf $S$,

Zinkernagel MS. $\mathrm{Br} \mathrm{J}$

Ophthalmol 2016;100

365-370.

\section{ABSTRACT}

Aims To investigate the prognostic significance of macular capillary drop-out and previous panretinal laser photocoagulation in diabetic macular oedema treated with intravitreal ranibizumab.

Methods Retrospective observational case series. Treatment-naive patients with diabetic macular oedema that had been treated with intravitreal ranibizumab as per the RESTORE study protocol for at least 12 months were included. Some patients $(n=15)$ had previous panretinal laser photocoagulation. Best-corrected visual acuity and central retina thickness were recorded monthly. The foveal avascular zone and the perifoveal capillaries were quantitatively and qualitatively assessed on fluorescein angiography on two occasions during the observational period.

Results From the 46 eyes (46 patients) in this study, $13(28 \%)$ had evidence of perifoveal capillary drop-out. Central retinal thickness was significantly thinner at baseline ( $p=0.02$ ) and throughout the study period in these eyes compared with those with normal perifoveal capillaries. Both groups responded with a significant gain of best-corrected visual acuity to ranibizumab treatment $(7.6 \pm 3.3$ and $6.3 \pm 1.3$ ETDRS letters, respectively). Eyes with previous panretinal laser photocoagulation displayed a comparable final outcome regarding function and morphology, requiring a similar intensity of intravitreal injections.

Conclusions Perifoveal capillary drop-out did not limit the gain of visual acuity from intravitreal ranibizumab treatment. The reduction of central retina thickness was similar to that seen in eyes with normal perifoveal capillaries. Central retinal thickness in eyes with perifoveal capillary drop-out was generally reduced. However, this did not affect their benefit from treatment. Ranibizumab did not increase the amount of perifoveal capillary loss.

\section{INTRODUCTION}

Diabetic retinopathy is the most common microvascular pathology in patients with diabetes mellitus, and is the leading cause of blindness in working aged adults in the industrialised world. Among patients with diabetic retinopathy, diabetic macular oedema (DMO) is the most frequent cause of visual impairment. It is an advanced visionlimiting complication that affects nearly $30 \%$ of patients who have suffered from diabetes mellitus for at least 20 years. $^{2}$

Prolonged hyperglycaemia, leading to chronic retinal hypoxia, is the major driving force in the development of DMO, a microvascular complication. The pathogenic mechanisms include thickening of the basal membrane of retinal capillaries and loss of perivascular pericytes. Chronic retinal microvascular damage and hypoxia result in elevation of intraocular levels of vascular endothelial growth factor A (VEGF), a molecule that greatly increases the permeability of blood vessels. ${ }^{3}$ Blockage of VEGF signalling has been shown to restore macular anatomy and considerably improve vision in several large randomised clinical trials. Ranibizumab (Genentech, South San Francisco, California, USA, and Novartis Schweiz, Basel, Switzerland), a monoclonal antibody fragment that binds to all isoforms of VEGF-A, has been shown to increase best-corrected visual acuity (BCVA) significantly by $11-12.5$ letters at month 24 and decrease central retinal thickness (CRT) when injected monthly as in the RISE and RIDE trials. ${ }^{4}$ Because VEGF is also an important constitutional trophic factor that is involved in endothelial proliferation and neovascularisation, ${ }^{5}$ as well as retinal collateral formation, ${ }^{6}$ and has been shown to increase blood flow in cerebral ischaemia, ${ }^{7}$ concerns have been articulated that the elimination of VEGF could compromise retinal capillaries and worsen ischaemia. However, despite some case reports showing increased capillary loss after intravitreal anti-VEGF treatment for $\mathrm{DMO},{ }^{8}$ a recent analysis of data from the RIDE and RISE trials suggested that monthly injections of ranibizumab might actually slow down the natural progression of retinal non-perfusion. ${ }^{9}$ Given the pivotal role of VEGF in the formation and exacerbation of DMO, it was our aim to investigate whether capillary loss alters the benefit from pro re nata treatment, and whether previous panretinal laser photocoagulation (PRP) might enhance the effect of anti-VEGF treatment.

\section{MATERIALS AND METHODS}

This was a single-centre retrospective observational case series. Investigators adhered to the tenets of the Declaration of Helsinki and Institutional Review Board/Ethics Committee approval was obtained for data collection and analysis (KEK-Nr. 093/13). The need for written consent from patients was waived because of the retrospective nature of the study. Study data were collected and managed using REDCap (Research Electronic Data Capture) hosted at our institution. ${ }^{10}$ REDCap is a secure, web-based application designed to support data management for research studies.

\section{Participants}

Adult patients with type 1 or 2 diabetes mellitus with centre-involving DMO, diagnosed with spectral 
domain optical coherence tomography (SD-OCT) (Heidelberg Spectralis, Heidelberg Engineering, Heidelberg, Germany) and fluorescein angiography, were assessed in this study (table 1).

Only treatment-naive eyes with at least 1 year of follow-up were included from the Retina Service of the Department of Ophthalmology at the University Hospital Bern, Switzerland. Exclusion criteria were any of the following: uncontrolled glaucoma, previous pars plana vitrectomy, evidence of focal macular laser or a history of intravitreal steroids, intraocular surgery or capsulotomy during the observational period. The loading protocol was identical for all patients and consisted of three monthly intravitreal injections of ranibizumab $0.5 \mathrm{mg} / 0.05 \mathrm{~mL}$, followed by monthly assessments including SD-OCT and further intravitreal ranibizumab as needed. Macular rescue laser was not used. The retreatment decision was based on the BCVA as per RESTORE study criteria. ${ }^{11}$

\section{Image acquisition}

SD-OCT scans were acquired using an established protocol consisting of both a crosshair and a volume scan. The volume scan, covering $20^{\circ} \times 20^{\circ}$, comprised 49 parallel B-scans separated by $120 \mu \mathrm{m}$, whereby each B-scan was the average of nine frames (automated real-time repetition rate $=9$ ), each consisting of 512 A-scans. CRT was determined automatically by the software (Heidelberg Eye Explorer, version 1.7.1.0, Heidelberg Engineering, Heidelberg, Germany) as the distance from the internal limiting membrane of the retina to the outer border of the retinal pigment epithelium.

For fluorescein angiography, $5 \mathrm{~mL}$ of $10 \%$ sodium fluorescein (Fluoresceine 10\% Faure 0.5 g/5 mL, Novartis Pharma Schweiz, Rotkreuz, Switzerland) was injected intravenously and images were obtained with a confocal scanning laser ophthalmoscope (Spectralis HRA, Heidelberg Engineering, Heidelberg, Germany).

\section{Quantification of central capillary loss and peripheral diabetic retinopathy severity}

The foveal avascular zone diameter, the perifoveal capillaries and the severity of diabetic retinopathy were independently assessed on fluorescein angiography by two fellowship-trained retina specialists (AE, MSZ) on two occasions during the observational period. Capillary loss in the central and the four inner subfields of the standard ETDRS grid $(1,3,6 \mathrm{~mm})$ was graded on early-phase fluorescein angiogram images as per the method described in the ETDRS Report Number $11 .^{12}$ In brief, a grading was assigned to each of the five subfields based on the standard photographs $1 \mathrm{~A}$ and 2 included in the aforementioned original publication. The grading steps for capillary loss were 0 (absent), 1 (questionable), 2 (definitely present), 3 (moderate) and 4 (severe). The maximum grade of capillary loss in any of the five subfields was then assigned as the final grade to the eye evaluated. Inter-rater agreement as quantified by Cohen's kappa was $0.91(95 \%$ CI 0.82 to 1.00$)$. If the grading differed

Table 1 Demographics of patients at baseline

\begin{tabular}{lc}
\hline Mean age, years (SD) & $63.5(12.3)$ \\
Male, $\mathrm{n}(\%)$ & $33(69)$ \\
Mean HbA1c, \% (SD) & $7.7(1.5)$ \\
Mean systolic blood pressure, mm Hg (SD) & $136(15)$ \\
Mean diastolic blood pressure, $\mathrm{mm} \mathrm{Hg}(\mathrm{SD})$ & $76(9)$ \\
Mean arterial blood pressure, mm Hg (SD) & $96(10)$ \\
Mean perifoveal capillary drop-out grading (SD) & $1.5(1.1)$ \\
\hline
\end{tabular}

Table 2 Diabetic Retinopathy Severity Score (DRSS) of patients without previous PRP at baseline

\begin{tabular}{llc}
\hline Diabetic retinopathy severity & DRSS level & $\mathbf{n}(\%)$ \\
\hline Absent & 10 & $1(3.1)$ \\
Microaneurysms only & 20 & $2(6.3)$ \\
Mild NPDR & 35 & $14(43.8)$ \\
Moderate NPDR & 43 & $5(15.6)$ \\
Moderately severe NPDR & 47 & $2(6.3)$ \\
Severe NPDR & 53 & $4(12.5)$ \\
Mild PDR & 61 & $2(6.3)$ \\
Moderate PDR & 65 & $2(6.3)$ \\
\hline NPDR, non-proliferative diabetic retinopathy; PDR, proliferative diabetic retinopathy; \\
PRP, panretinal laser photocoagulation.
\end{tabular}

between assessors, the case was reviewed and discussed to reach a common conclusion on the final grade to be assigned. Capillary drop-out was deemed present in eyes with a capillary loss grading $>1$.

Similarly, in eyes without previous PRP, diabetic retinopathy severity was classified into eight levels (table 2) according to the ETDRS Report Number 12 protocol with slight modifications, ranging from absent retinopathy (Level 10) to moderate proliferative diabetic retinopathy (Level 65). ${ }^{13}$

For quantification of the size of the foveal avascular zone, the diameter of the outline of the foveal avascular zone was determined using the circle measurement tool available in the Heidelberg Eye Explorer software (see online supplementary figure for schematic illustration).

\section{Statistical analysis}

The last observation carried forward approach was used to substitute missing BCVA data at the final visit. Statistical analysis was performed using a standard software package (Prism 6, GraphPad Software, La Jolla, California, USA) and R for Mac OS X (R 3.1.2 GUI 1.65 Mavericks build). Unless indicated otherwise, results are given as mean \pm SEM in the text.
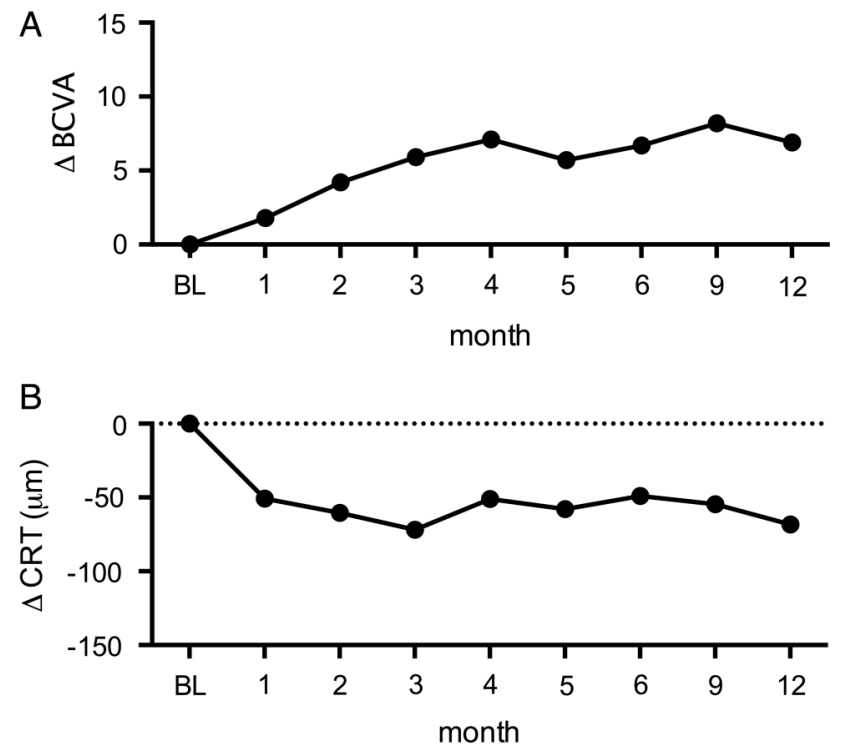

Figure 1 Best-corrected visual acuity (BCVA) and retinal thickness over 12 months. (A) Mean change in BCVA ETDRS letter score from baseline $(\mathrm{BL})$ to month 12 . (B) Mean change in central retinal thickness (CRT) from BL to month 12. 
Table 3 Comparison between eyes with and without capillary drop-out

\begin{tabular}{llllll}
\hline & Subjects with PRP & IVTs per year $\left(\right.$ mean $_{ \pm}$SD) & Baseline BCVA (mean \pm SD) & CRT baseline (mean \pm SD) & Retinal fluid at final visit \\
\hline No capillary drop-out $(n=27)$ & 12 & $5.9 \pm 2.1$ & $62.6 \pm 16.3$ & $433 \pm 108 \mu \mathrm{m}$ & $88 \%$ \\
Capillary drop-out $(\mathrm{n}=13)$ & 8 & $5.0 \pm 2.0$ & $60.1 \pm 14.0$ & $349 \pm 91 \mu \mathrm{m}$ & $90 \%$ \\
$\mathrm{p}$ Value & $0.258^{*}$ & $0.203 \dagger$ & $0.641 \dagger$ & $0.020 \dagger$ & $1.0^{*}$ \\
\hline
\end{tabular}

*Pearson's $\chi^{2}$ test with Yates' continuity correction. + Two-sided unpaired Student's $t$ test.

BCVA, best-corrected visual acuity; CRT, central retinal thickness; IVT, intravitreal treatment; PRP, panretinal laser photocoagulation.

Depending on the distribution of the statistical sample, data were analysed using the Pearson's $\chi^{2}$ test and the paired or unpaired Student's t test with correction for multiple comparisons as needed. To rule out interference between the main outcomes, diabetic retinopathy severity and capillary drop-out, two-way ANOVA testing was conducted to assess the effects of Diabetic Retinopathy Severity Score (DRSS) score and capillary drop-out grading on final BCVA and the average number of intravitreal ranibizumab treatments per year. We did not find any interaction between the capillary drop-out grading and the DRSS score, and the null hypothesis (independence) was not rejected in the Fisher's exact test $(\mathrm{p}=0.408)$.
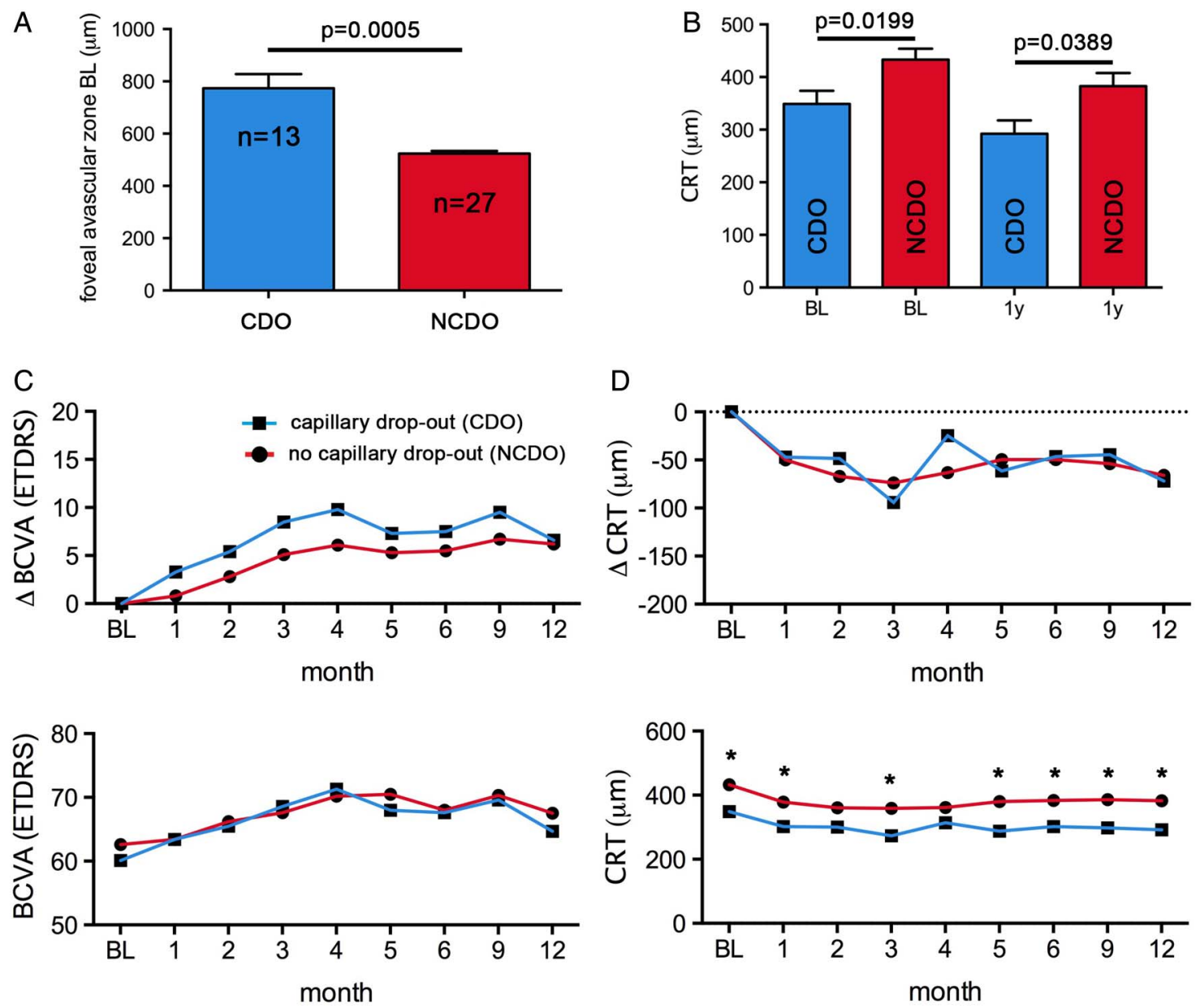

Figure 2 Outcomes for eyes with and without perifoveal capillary drop-out (CDO) of the macula. (A) Diameter of foveal avascular zone at baseline $(\mathrm{BL})$ in eyes with perifoveal CDO (capillary loss grading $>1$ ) and eyes with normal appearance of the perifoveal capillaries (NCDO; capillary loss grading $\leq 1)$. (B) Central retinal thickness (CRT) at BL and 1 year after initiation of intravitreal treatment with ranibizumab. CRT was consistently lower in eyes with CDO. (C) Mean improvement in best-corrected visual acuity (BCVA) and mean absolute ETDRS letters scores from BL to month 12 for both groups. (D) Mean decrease and mean absolute CRT for both groups from BL to month 12 ( ${ }^{*} p<0.05$; Multiple t tests using the Holm-Sidak method to correct for multiple testing; error bars in A and B represent SEM).

A $\mathrm{p}$ value of less than 0.05 was considered statistically significant.

\section{RESULTS}

Forty eight patients that had started intravitreal ranibizumab treatment for DMO between October 2010 and April 2013 at the Retina Service of the Department of Ophthalmology at the University Hospital Bern had data for 12 months. Two patients with a foveal avascular zone diameter of more than $1500 \mu \mathrm{m}$ were excluded from the analysis to avoid distortion of the results by macular ischaemia, which is usually associated with poor visual prognosis. The mean age of the patients at the time of

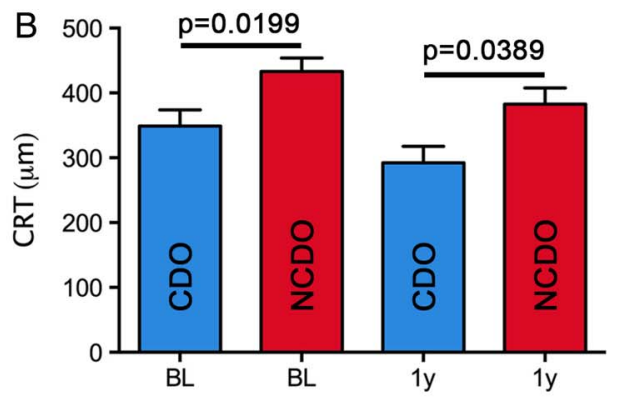



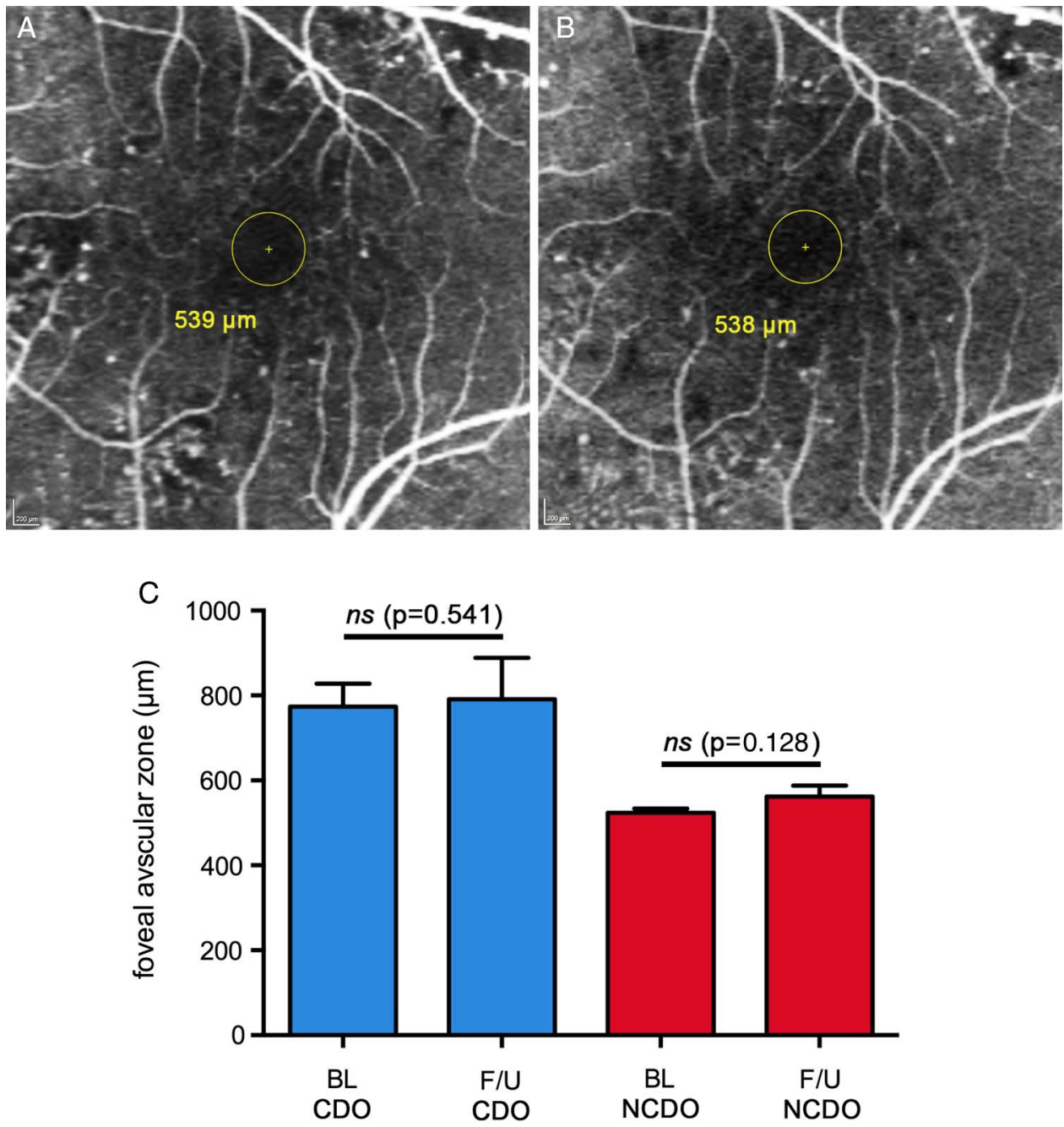

Figure 3 Progression analysis of perifoveal capillary drop-out (CDO). Representative photographs of a macula at baseline (BL) (A) and after 17 months of treatment (B) with a constant CDO grading. The patient received in total five intravitreal ranibizumab injections. His diabetic retinopathy was graded as 'severe non-proliferative' (level 53) and the CDO grading assigned was 'questionable' (grade 1). (C) Diameter of foveal avascular zone at BL and follow-up (F/U) visit on fluorescein angiography for eyes with CDO and normal perifoveal capillaries (NCDO). The mean interval between BL and follow-up fluorescein angiography was 13 months. The change of the foveal avascular zone diameter was not significant (paired Student's t test; error bars represent SEM).

diagnosis was 63.7 years (range $32-89$ years). The baseline characteristics of the study population are summarised in table 1.

The mean BCVA at baseline was 61.4 \pm 2.2 ETDRS letters and increased to $68.3 \pm 2.1$ letters at month $12(\mathrm{p}=0.0002)$. CRT was $398 \pm 15 \mu \mathrm{m}$ at baseline and $349 \pm 19 \mu \mathrm{m}$ at month 12 $(p=0.0001)$. On average, patients received $5.8 \pm 0.3$ intravitreal injections of ranibizumab (range 3-10) in the first year of treatment. The changes in BCVA and CRT in our patient cohort during the first 12 months were similar to other published data and are summarised in figure 1 .

While all 46 eyes had intraretinal fluid (IRF) at baseline, only four eyes (8.7\%) had concomitant subretinal fluid (SRF) at that time. While after the three initial monthly injections SRF had disappeared in all of these eyes, IRF persisted in 38 eyes (83\%) after the initiation phase. In 31 eyes $(67 \%)$ IRF was present at every monthly visit throughout the observational period.

\section{Perifoveal capillary drop-out, CRT and BCVA}

The findings from the comparison between eyes with capillary drop-out and eyes with normal macular capillaries are summarised in table 3 .
The diameter of the foveal avascular zone at baseline was significantly larger in eyes with perifoveal capillary drop-out $(p=0.0005$; unpaired $t$ test; figure $2 \mathrm{~A})$. The initial CRT in the capillary drop-out group was lower than in the eyes with unaffected perifoveal capillary structure, and this difference persisted throughout the observational period (figure $2 \mathrm{~B}$ ). The gain in visual acuity was of similar magnitude for eyes with and without perifoveal capillary drop-out $(7.6 \pm 3.3$ and $6.3 \pm 1.3$ ETDRS letters, respectively; figure 2C). CRT reduction was comparable in both groups (figure 2D). On fluorescein angiography, there was no evidence of deterioration or improvement of the vascular structures in the central subfield and the inner ring of the ETDRS grid during the average 13 months period between examinations (figure 3). Eyes with perifoveal capillary drop-out needed by trend fewer intravitreal treatments than eyes with unremarkable perifoveal capillaries (table 3).

\section{PRP, CRT and BCVA}

Twenty four eyes in the study population had advanced disease requiring peripheral photoablation. While 15 eyes had received PRP prior to anti-VEGF therapy for DMO (last treatment 
A
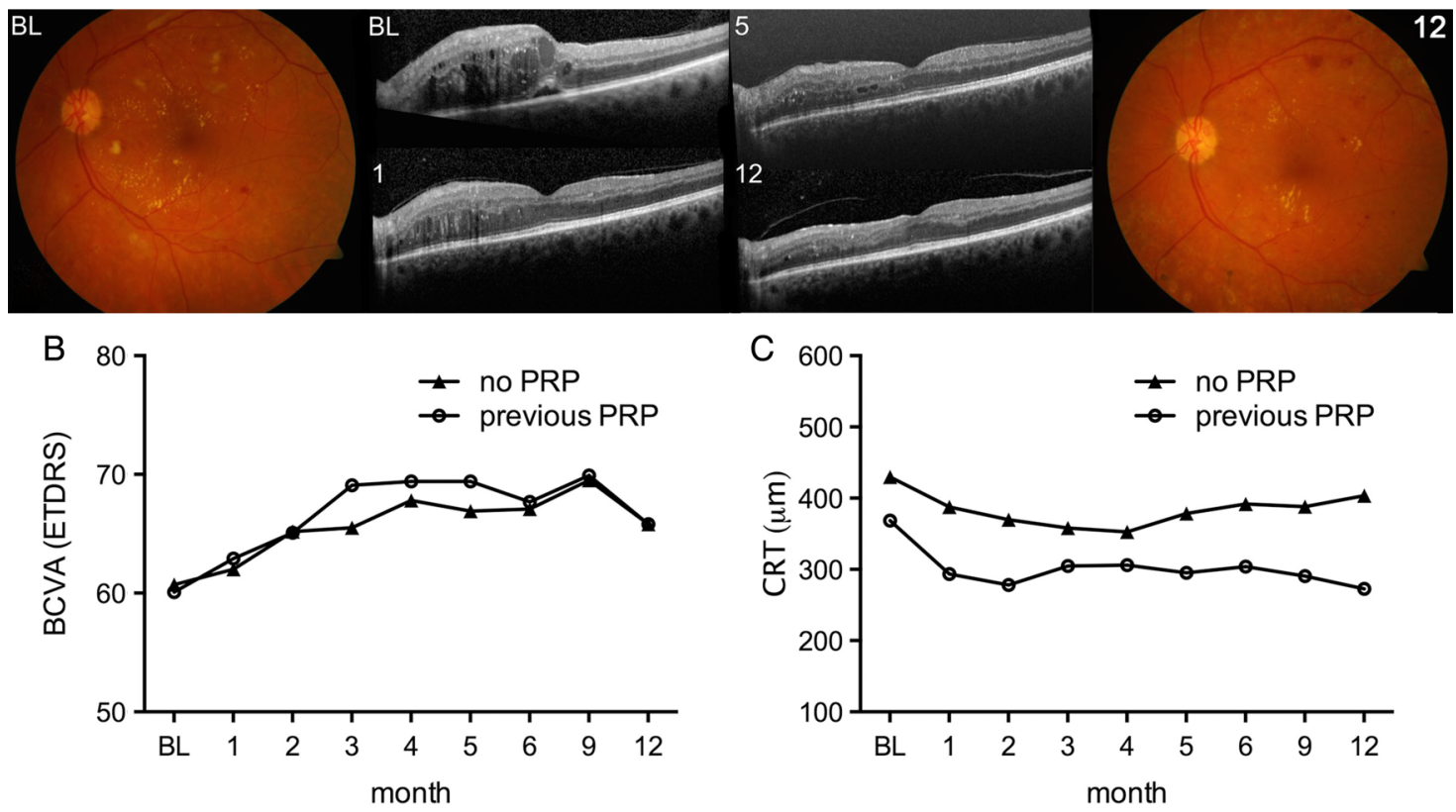

Figure 4 Outcome analysis according to panretinal laser photocoagulation (PRP) status. (A) Colour fundus photographs at baseline (BL) and month 12 as well as corresponding optical coherence tomography scans at BL, months 1, 5 and 12. (B) Best-corrected visual acuity (BCVA) letter score from BL to month 12 for eyes without (no PRP) and with previous PRP. (C) Corresponding graph for central retinal thickness (CRT). Differences were not statistically significant at any time point (multiple $t$ tests using the Holm-Sidak method to correct for multiple comparisons).

$31.0 \pm 10.1$ months (range 6 weeks-12 years) before commencement of intravitreal ranibizumab injections), 9 eyes received concomitant panretinal laser treatment during the observational period. These nine eyes were excluded for the analyses investigating the effects of previous PRP. Analysing the influence of previous PRP on BCVA, CRT and the number of injections needed, eyes with previous PRP $(n=15)$ received by trend more injections than eyes without previous peripheral laser treatment $(6.3 \pm 0.6$ vs $5.2 \pm 0.4)$. However, the difference was not significant $(p=0.187$; unpaired t test with Welch's correction). The benefit in ETDRS letter gain (figure 4B) was similar and the reduction in CRT was comparable in both groups (figure 4C).

\section{DISCUSSION}

The mean gain in BCVA $(6.9 \pm 1.4$ letters $)$ from baseline to month 12 in this series was similar to results reported in the RESTORE study $(6.8 \pm 0.8$ letters), which used the same retreatment criteria and studied a sample $(n=116$ in the ranibizumab group) with comparable baseline mean BCVA (64.8 \pm 0.9$)$. The mean number of intravitreal ranibizumab injections needed to achieve this result in our cohort (5.8 injections; median (range) :6 (3-10)) was lower than in the RESTORE study (7.0 injections; median (range) :7 (1-12); $\mathrm{p}=0.016)$. This dissimilarity in the treatment intensity might be explained by the probably somewhat greater macular thickness of subjects included in the RESTORE study, which used time-domain OCT (Stratus, Carl Zeiss, Meditec, Dublin, California, USA). In the current study SD-OCT (Spectralis HRA+OCT, Heidelberg Engineering, Heidelberg, Germany) was used, which has been reported to measure macular thickness systematically $77 \mu \mathrm{m}$ thicker than the Stratus OCT. ${ }^{14}$ Hence, the mean CRT reported in the RESTORE study of $427 \pm 118$ (SD) $\mu \mathrm{m}$ should be corrected to $504 \mu \mathrm{m}$ for comparison, which is significantly different from the CRT of 398 \pm 105 (SD) $\mu \mathrm{m}$ in the sample of the present study $(\mathrm{p}<0.0001)$.
In contrast to exudative age-related macular degeneration, the persistence of IRF or SRF is generally not considered imminently disconcerting in DMO. Nevertheless, the anatomical location of fluid collections seems to have an effect on function. Photoreceptor outer segment disturbance has been linked to impaired BCVA in DMO, ${ }^{15}$ and reduced retinal sensitivity was found in the presence of SRF using microperimetry. ${ }^{16}$ There is debate about the significance of persistent fluid on function and prognostic implications for visual acuity. Excessive suppression of VEGF, which at moderate levels is physiologically present in the healthy retina, ${ }^{17} 18$ might harm the choroid and accelerate progression of atrophy. ${ }^{19}$ In the pivotal RESTORE study, retinal thickness and OCT-based assessment of the retina were not included in the retreatment criteria that guided the administration of intravitreal ranibizumab. ${ }^{11}$ The current study applied the RESTORE criteria. The visual acuity gain for eyes without recurrent or persistent retinal fluid was slightly higher in the subgroup analysis. IRF was very common and in two-thirds of eyes was present at every monthly visit, which, however, does not exclude the possibility that temporary resolution might have occurred at early time points after treatment. In contrast, SRF was found in less than $10 \%$ of eyes at baseline, and had resolved completely in all eyes after the three initial monthly ranibizumab injections. SRF was always seen in conjunction with IRF and neither previous PRP nor the presence of capillary drop-out had an influence on the occurrence of neurosensory detachments.

Concerns have also been raised as to whether chronic use of anti-VEGF might further compromise retinal perfusion in eyes with vascular disease. ${ }^{20}$ In the present study perifoveal capillary loss did not negatively affect the benefit from ranibizumab treatment despite the fact that such retinas were consistently thinner, and we did not find any evidence for worsening of capillary drop-out.

At first glance counterintuitive is the observation that eyes with previous PRP received by trend more intravitreal injections 
than eyes without previous peripheral laser. VEGF, a major driving force of macular oedema is known to be elevated in eyes with diabetic retinopathy ${ }^{3}$ and there is debate about the role of peripheral laser ablation in preventing or reducing DMO. ${ }^{21}$ Eyes that have had previous laser presumably have more advanced peripheral microvascular disease, which in turn increases the risk of diffuse macular oedema. ${ }^{22}$

In conclusion, perifoveal capillary drop-out did not limit gain of visual acuity from intravitreal ranibizumab treatment. The reduction of CRT was similar as in eyes with unremarkable perifoveal capillaries. Interestingly, CRT in eyes with perifoveal capillary drop-out was generally reduced. Ranibizumab did not increase the amount of perifoveal capillary loss and the presence of peripheral laser scars from previous PRP did not reduce the treatment intensity needed to achieve treatment goals. The role of peripheral laser photoablation in the context of anti-VEGF treatment for DMO in patients with nonproliferative disease needs to be elucidated in larger prospective trials.

Contributors AE: design and conduct of the study; collection, management, analysis, and interpretation of the data; preparation, review and approval of the manuscript. SW: data acquisition; review and approval of the manuscript; administrative and technical support; supervision. MSZ: design and conduct of the study; data acquisition; data analysis and interpretation; preparation, review and approval of the manuscript; administrative and technical support; supervision.

\section{Funding None.}

Competing interests $A E$ received honoraria from Bayer for lectures. SW received financial support from Novartis, Alcon, Roche, Bayer, Optos and Heidelberg Engineering. MSZ received financial support from Novartis, Bayer and Heidelberg Engineering.

Ethics approval Ethics Committee of the Canton of Bern (KEK-Nr. 093/13).

Provenance and peer review Not commissioned; externally peer reviewed.

Data sharing statement All data pertaining to this study are included in the manuscript.

Open Access This is an Open Access article distributed in accordance with the Creative Commons Attribution Non Commercial (CC BY-NC 4.0) license, which permits others to distribute, remix, adapt, build upon this work non-commercially, and license their derivative works on different terms, provided the original work is properly cited and the use is non-commercial. See: http://creativecommons.org/ licenses/by-nc/4.0/

\section{REFERENCES}

1 Congdon N, O'Colmain B, Klaver CC, et al. Causes and prevalence of visual impairment among adults in the United States. Arch Ophthalmol 2004;122:477-85.

2 Klein R, Knudtson MD, Lee KE, et al. The Wisconsin Epidemiologic Study of Diabetic Retinopathy XXIII: the twenty-five-year incidence of macular edema in persons with type 1 diabetes. Ophthalmology 2009;116:497-503.
3 Aiello LP, Avery RL, Arrigg PG, et al. Vascular endothelial growth factor in ocular fluid of patients with diabetic retinopathy and other retinal disorders. $N$ Engl J Med 1994;331:1480-7.

4 Nguyen QD, Brown DM, Marcus DM, et al. Ranibizumab for diabetic macular edema: results from 2 phase III randomized trials: RISE and RIDE. Ophthalmology 2012;119:789-801.

5 Esser S, Lampugnani MG, Corada M, et al. Vascular endothelial growth factor induces VE-cadherin tyrosine phosphorylation in endothelial cells. J Cell Sci 1998;111(Pt 13):1853-65.

6 Clayton JA, Chalothorn D, Faber JE. Vascular endothelial growth factor-A specifies formation of native collaterals and regulates collateral growth in ischemia. Circ Res 2008; 103:1027-36.

7 Zechariah A, ElAli A, Doeppner TR, et al. Vascular endothelial growth factor promotes pericyte coverage of brain capillaries, improves cerebral blood flow during subsequent focal cerebral ischemia, and preserves the metabolic penumbra. Stroke 2013;44:1690-7.

8 Goel N, Kumar V, Ghosh B. Ischemic maculopathy following intravitreal bevacizumab for refractory diabetic macular edema. Int Ophthalmol 2011;31:39-42.

9 Campochiaro PA, Wykoff CC, Shapiro $\mathrm{H}$, et al. Neutralization of vascular endothelial growth factor slows progression of retinal nonperfusion in patients with diabetic macular edema. Ophthalmology 2014;121:1783-9.

10 Harris PA, Taylor R, Thielke R, et al. Research electronic data capture (REDCap)-a metadata-driven methodology and workflow process for providing translational research informatics support. J Biomed Inform 2009;42:377-81.

11 Mitchell P, Bandello F, Schmidt-Erfurth U, et al. The RESTORE study: ranibizumab monotherapy or combined with laser versus laser monotherapy for diabetic macular edema. Ophthalmology 2011;118:615-25.

12 [No authors listed]. Classification of diabetic retinopathy from fluorescein angiograms. ETDRS report number 11. Early Treatment Diabetic Retinopathy Study Research Group. Ophthalmology 1991;98:807-22.

13 [No authors listed]. Fundus photographic risk factors for progression of diabetic retinopathy. ETDRS report number 12 . Early Treatment Diabetic Retinopathy Study Research Group. Ophthalmology 1991;98:823-33.

14 Wolf-Schnurrbusch UE, Ceklic L, Brinkmann CK, et al. Macular thickness measurements in healthy eyes using six different optical coherence tomography instruments. Invest Ophthalmol Vis Sci 2009;50:3432-7.

15 Alasil T, Keane PA, Updike JF, et al. Relationship between optical coherence tomography retinal parameters and visual acuity in diabetic macular edema. Ophthalmology 2010;117:2379-86.

16 Deak GG, Bolz M, Ritter M, et al. A systematic correlation between morphology and functional alterations in diabetic macular edema. Invest Ophthalmol Vis Sci 2010;51:6710-14.

17 Maharaj AS, D'Amore PA. Roles for VEGF in the adult. Microvasc Res 2007;74:100-13.

18 Saint-Geniez M, Maharaj AS, Walshe TE, et al. Endogenous VEGF is required for visual function: evidence for a survival role on Muller cells and photoreceptors. PloS One 2008;3:e3554.

19 Lois N, McBain V, Abdelkader E, et al. Retinal pigment epithelial atrophy in patients with exudative age-related macular degeneration undergoing anti-vascular endothelial growth factor therapy. Retina 2013;33:13-22.

20 Salam A, DaCosta J, Sivaprasad S. Anti-vascular endothelial growth factor agents for diabetic maculopathy. Br J Ophthalmol 2010;94:821-6.

21 Gaucher D, Fortunato $P$, LeCleire-Collet $A$, et al. Spontaneous resolution of macular edema after panretinal photocoagulation in florid proliferative diabetic retinopathy. Retina 2009;29:1282-8.

22 Lopes de Faria JM, Jalkh $A E$, Trempe $C L$, et al. Diabetic macular edema: risk factors and concomitants. Acta Ophthalmol Scand 1999;77:170-5. 